\title{
What Have We Learned from the European Society of Cardiology 2019 Guidelines on Supraventricular Tachycardia
}

\author{
Erdi Babayiğit ${ }^{a} \quad$ Taner Ulus $^{b} \quad$ Bülent Görenek ${ }^{b}$

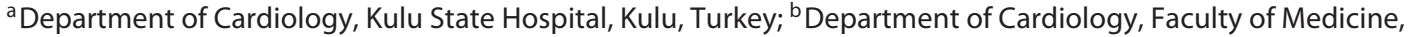 \\ Eskişehir Osmangazi University, Eskişehir, Turkey
}

\section{Keywords}

Supraventricular tachycardia $\cdot$ Arrhythmia $\cdot$ European Society of Cardiology Supraventricular Tachycardia Guidelines · Electrophysiology

\begin{abstract}
Supraventricular tachycardias (SVTs) are common arrhythmic conditions in clinical practice. Increased knowledge and experience on SVTs and some unclear situations in clinical practice led the European Society of Cardiology (ESC) team to write a new guideline. In this review, we touch upon the important points in the new ESC 2019 SVT guidelines and present changing approaches and suggestions. By providing a general review on SVTs, we also mention the basic mechanism, epidemiology, and clinical presentation of SVTs, approaching narrow and wide QRS tachycardias, SVTs in special patient groups, and treatment of SVTs.
\end{abstract}

(C) 2020 S. Karger AG, Basel

\section{Introduction}

Supraventricular arrhythmias are common and various arrhythmic conditions that may come across as acute or chronic presentations. Increasing clinical knowledge and experience in recent years, especially with electrophysiologic studies and ablation procedures, has changed

karger@karger.com

(c) 2020 S. Karger AG, Basel

www.karger.com/crd

Karger! the diagnostic, risk stratification, and treatment approaches in clinical practice. In this context, the European Society of Cardiology (ESC) Guidelines on Supraventricular Tachycardia (SVT) were published in 2003 [1], then the US guidelines on SVT were published in 2015 [2], and the ESC team prepared a new guideline on SVT in 2019 [3]. In this paper, we will try to address the new and emerging points in the clinical approach with the recent guidelines.

\section{Definitions, Classifications, and Mechanisms of SVTs}

SVT is a term used to describe arrhythmias involving the His bundle or above tissues where atrial and/or ventricular rates are $>100 \mathrm{bpm}$ at rest [2]. In new ESC SVT guidelines, SVTs are conventionally classified into three major groups: atrial tachycardia (AT), atrioventricular (AV) junctional tachycardia, and AV re-entrant tachycardia (AVRT). Although atrial fibrillation (AF) is a supraventricular arrhythmia, it has not been included in the SVT guidelines.

There are three different mechanisms considered in the occurrence of SVTs. The first mechanism, named reentry, occurs by abnormal propagation of stimuli on the basis of different excitability and refractoriness of myocardial tissue regions. Abnormal or enhanced automaticity is the mechanism whereby arrhythmia can occur with 


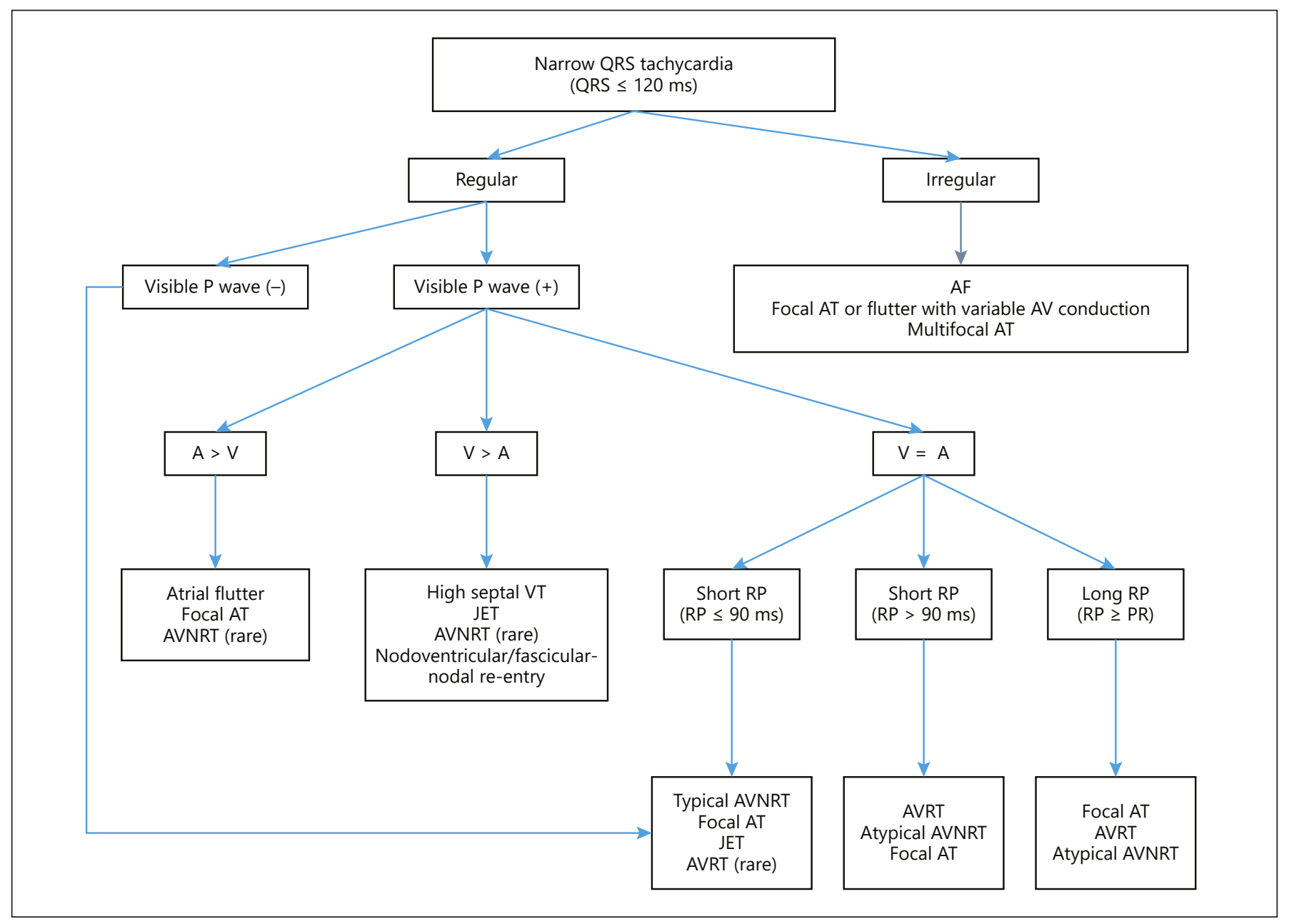

Fig. 1. Differential diagnosis of narrow QRS tachycardia. A, atrial rate; AF, atrial fibrillation; AT, atrial tachycardia; AV, atrioventricular; AVNRT, atrioventricular nodal re-entrant tachycardia; AVRT, atrioventricular reentrant tachycardia; JET, junctional ectopic tachycardia; PR, PR interval; RP, RP interval; V, ventricular rate; VT, ventricular tachycardia.

similar mechanism to physiologic pacemaker activity. The triggered activity is the third mechanism, which is caused by early or delayed after-depolarisations.

\section{Epidemiology of SVTs}

The prevalence of SVT is $2.25 / 1,000$ persons and the incidence is $35 / 100,000$ person-years. Women tend to have twice the risk of SVT than men. After the age of 65 years, the risk of developing SVT is five times greater than in young individuals. In the young population, SVTs are generally lone paroxysmal, and patients have faster heart rates [4]. The annual risk of sudden cardiac death is $0.01 \%$ per patient-year by the age of 15 years [5].

ESC 2019 Supraventricular Tachycardia Guidelines: Review
After AF, the most commonly treated arrhythmia with catheter ablation is AV nodal re-entrant tachycardia (AVNRT), followed by atrial flutter and AVRT [6-8]. While AVNRTs are more common in women, AVRTs occur more frequently in men [3]. In pregnancy, SVT attacks are more frequent in women with pre-existing SVT [9]. The prevalence of Wolff-Parkinson-White (WPW) syndrome in the general population is about $0.15-0.25 \%$ [10]. Atrial flutter incidence is $88 / 100,000$ person-years in the US, and it tends to be more common in men after adjustment for age. Atrial flutter tends to be observed in smokers and subjects who have chronic pulmonary diseases, those with longer PR interval, and patients with a history of myocardial infarction or heart failure (HF) [11]. 


\section{Clinical Findings and Initial Evaluation}

The rapid onset of tachycardia, the patient's age at onset, and the duration of symptoms affect the development of clinical signs [3]. Rapid rhythms lead to more symptoms. An onset in the teenage years or younger is less likely to be AT or AF. A long clinical history refers to the re-entrant mechanism $[12,13]$. At an older age, symptoms can be more serious, such as presyncope and syncope [13]. SVTs are generally not life-threatening, but some specific situations (in patients with WPW syndrome and AF or after atrial switch operation) can cause sudden cardiac death $[14,15]$.

If the onset and termination of the arrhythmia is sudden, it is associated with a change in position, and if it is regular, it indicates a re-entrant mechanism [3]. An electrocardiography (ECG) recording during tachycardia is very useful. Baseline ECG and echocardiography are recommended for initial evaluation. Myocardial ischaemia evaluation is recommended in patients with angina or significant risk factors for coronary artery disease [16].

\section{Differential Diagnosis}

\section{Narrow QRS Tachycardia ( $\leq 120 \mathrm{~ms})$}

The PR interval suddenly increases after an atrial ectopic beat in typical AVNRT while premature ventricular complexes are common triggers of atypical AVNRT. ATs may initiate with atrial ectopic beat without PR interval prolongation, and automatic focal ATs initiate with warm-up and end with cool-down phenomena [17]. AVRTs can be triggered with premature atrial or ventricular beats.

In recent guidelines, the differential diagnosis of narrow QRS tachycardia has been evaluated more comprehensively compared to the 2003 SVT guidelines. Atrial and ventricular rates were evaluated separately. The diagram for the differential diagnosis of narrow QRS tachycardia is shown in Figure 1.

Vagal manoeuvres and adenosine can help with the diagnosis and termination of arrhythmia. If arrhythmia terminates with a $\mathrm{P}$ wave after the last QRS complex, the arrhythmia is most likely AVRT and typical AVNRT, very unlikely AT. If SVT terminates with a QRS complex, it is most likely AT and possibly atypical AVNRT. Termination with a QRS complex is more common in AT and atypical AVNRT. In narrow QRS tachycardia, after adenosine injection, different responses can be seen. If adenosine is ineffective, the drug dose may be insufficient or it may be high septal ventricular tachycardia (VT). If gradual slowing then reacceleration occurs, it may be sinus tachycardia, automatic focal AT, or junctional ectopic tachycardia. If sudden termination occurs, it may be AVNRT, AVRT, sinus nodal re-entry, or triggered focal AT. If an atrial tachyarrhythmia persists with transient high-grade AV block after adenosine, it may be atrial flutter or micro-re-entrant focal AT [3]. Finally, electrophysiologic study manoeuvres and techniques can be applied for a definitive differential diagnosis.

\section{Wide QRS Tachycardia (>120 ms)}

Wide QRS tachycardia, which can be VT with a probability of $80 \%$, can also be observed with some supraventricular arrhythmias. Among the causes of wide QRS complex tachycardia, positive predictive values for VT increase up to $98-100 \%$ in patients with a history of myocardial infarction, congestive HF, or recent angina pectoris [18]. One study declared that age $>35$ years has a $92 \%$ sensitivity for VT in wide QRS complex tachycardias [18]. SVT with bundle branch blocks with a probability of $15 \%$ can occur during tachycardia and pre-excited SVT which occurs when the tachycardia circuit conducts as anterograde over an accessory pathway (AP). Drugs or electrolyte disturbances may widen QRS.

ECG features suggesting VT are presence of AV dissociation $(\mathrm{V}>\mathrm{A})$, fusion/capture beats, negative concordance in precordial leads, RS duration in any lead $>100$ $\mathrm{ms}$, presence of initial $\mathrm{R}$, initial $\mathrm{R}$ or Q wave $>40 \mathrm{~ms}$, a notch of a predominantly negative complex in lead aVR, and presence of the northwest axis. In right bundle branch morphology, monophasic R, qR complex, broad $\mathrm{R}$ wave ( $>40 \mathrm{~ms}$ ), a double-headed $\mathrm{R}$ wave with the first peak taller than the later in V1, and an R/S ratio $<1$ in V6 are in favour of VT. In left bundle branch morphology, broad R wave, notched-down stroke of the $S$ wave, delayed bottom point of the $\mathrm{S}$ wave in $\mathrm{V} 1$, and Q or QS wave in V6 are in favour of VT [3].

\section{Irregular Tachycardia}

Irregular ventricular rhythm is mostly observed in AF, multifocal AT, and focal AT or atrial flutter with variable ventricular conduction (Fig. 1). Polymorphic VT, rarely monomorphic VT, and junctional non-re-entrant tachycardia may also have irregular QRS. In pre-excited AF, irregularity of QRS complexes and QRS morphology variations result from the fusion of impulses from both AV node and AP with variable conductions. As a result, the width of the delta wave varies. 


\section{Acute Management without an Established Diagnosis}

\section{Regular Narrow QRS Tachycardia}

From non-drug-based approaches to intravenous drugs and electrical cardioversion, acute treatment recommendations for SVTs were prepared to make a quick and accurate decision. As a rule, immediate direct-current (DC) cardioversion is the first option in haemodynamically unstable patients (class I B).

As a first-line treatment in haemodynamically stable patients, vagal manoeuvres, preferably the Valsalva manoeuvre in the supine position with leg elevation, can be used (class I B). Carotid sinus massage should always be unilateral and limited to $5 \mathrm{~s}$ and should be avoided in patients with previous transient ischaemic attack or stroke or in patients with carotid bruits. Adenosine is the first drug which can be used in narrow QRS tachycardia (class I B). It delays AV conduction with a dose-related effect and can terminate the SVT. Doses start with $6 \mathrm{mg}$, then $12 \mathrm{mg}$ and $18 \mathrm{mg}$. The drug should be given quickly and carefully considering its side effects such as transient dyspnoea, facial flushing, chest pain, bradycardia, AF, and pre-excited atrial arrhythmias [19-21]. If adenosine is ineffective, intravenous verapamil, diltiazem, or beta-blockers such as intravenous metoprolol or esmolol can be used as a second choice (class IIa C). If there is haemodynamic instability, $\mathrm{HF}$ with reduced ejection fraction (HFrEF), suspicion of VT, or pre-excited AF, calcium channel blockers should be avoided. Verapamil and diltiazem had class I A recommendation and beta-blockers had class IIb $\mathrm{C}$ recommendation in previous guidelines [1]. In recent guidelines, verapamil and diltiazem are recommended with class IIa B and beta-blockers are recommended with class IIa C. If drug therapy fails to control or convert the tachycardia, synchronised DC cardioversion is recommended with class I B. Etripamil, a short-acting L-type calcium channel blocker administered intranasally and newly mentioned in the 2019 guidelines, has shown successful conversion rates of $65-95 \%$ from SVT to sinus rhythm [22]. Amiodarone and digoxin are no longer recommended.

\section{Regular Wide QRS Tachycardia}

In haemodynamically unstable patients with any persistent wide QRS tachycardia, such as hypotension, acutely altered mental status, chest pain, acute HF symptoms, or signs of shock, synchronised DC cardioversion should be performed (class I B).

Unlike in previous guidelines, vagal manoeuvres are recommended first with class I C in haemodynamically

ESC 2019 Supraventricular Tachycardia Guidelines: Review stable patients for providing insight into the mechanism responsible for arrhythmia in the 2019 guidelines. If SVT with aberrancy is definitely identified, then arrhythmia can be treated as SVT. Adenosine may be used for diagnosis and adenosine-sensitive VTs with class IIa C recommendation level, providing there is no pre-excitation on the resting ECG. This recommendation level was class IIb $\mathrm{C}$ in previous guidelines [1]. Adenosine can induce $\mathrm{AF}$ and occasionally may result in precipitated cardiac arrest in antidromic re-entrant tachycardia. If adenosine is ineffective, intravenous procainamide (class IIa B) or amiodarone (class IIb B) can be used for third-line pharmacologic therapy. Procainamide, amiodarone, sotalol, and DC cardioversion were recommended with class I B in previous guidelines [1]. In the PROCAMIO trial, procainamide was superior to amiodarone in termination of tachycardia within $40 \mathrm{~min}$ in patients with or without reduced left ventricular (LV) ejection fraction and stable wide QRS tachycardia, and it was associated with fewer major cardiac adverse events [23]. If these therapies fail to control or convert the tachycardia, synchronised DC cardioversion is recommended with class I B. If the mechanism of wide QRS tachycardia is not fully understood, then it should be treated as VT. Verapamil is not recommended in wide QRS tachycardia when the aetiology is unknown (class III B). Sotalol and lidocaine are not longer mentioned [3].

\section{Irregular QRS Tachycardia}

Irregular wide or narrow QRS complex rhythms are often observed as a result of AF. Rarely, polymorphic VT and extremely infrequent monomorphic VT may present as irregular wide QRS tachycardia. The differential diagnosis also includes proarrhythmia (torsades de pointes VT). Synchronised DC cardioversion should be performed for acute treatment of irregular pre-excited tachycardia when haemodynamic instability occurs.

\section{Specific Arrhythmias and Managements}

In the 2019 Guidelines atrial arrhythmias, AV junctional arrhythmias, and AV arrhythmias are evaluated separately.

\section{Atrial Arrhythmias}

Sinus Tachycardia. Sinus tachycardia is defined as a sinus rhythm $>100 \mathrm{bpm}$. In physiologic sinus tachycardia, there is an underlying physiologic (exercise, pregnancy) or medical condition with normal $\mathrm{P}$ wave morphol- 
ogy. Treatment of reversible causes is recommended in recent guidelines. (1) Inappropriate sinus tachycardia (IST) is defined as a sinus rhythm $>100 \mathrm{bpm}$ at rest or minimal activity with disproportionately faster than expected [24]. The diagnosis of IST is based on the exclusion of the other arrhythmias such as physiologic sinus tachycardia, postural orthostatic tachycardia syndrome, sinus re-entrant tachycardia, or focal AT from the superior part of the crista terminalis or right superior pulmonary veins. Reassurance, exercise training, volume expansion, and avoidance of cardiac stimulants are pre-drug treatment modalities. In the 2019 guidelines, ivabradine alone or in combination with a beta-blocker is recommended with class IIa B and beta-blockers are recommended with class IIa $\mathrm{C}$ in patients with symptomatic IST. The recommendations in previous guidelines were beta-blockers with class I C, verapamil and diltiazem with class IIa C, and catheter ablation with class IIb C [1]. Verapamil and catheter ablation are not longer mentioned [3]. (2) Sinus node re-entrant tachycardia (SNRT) is a re-entry circuit which involves the sinus node. The difference between SNRT and IST is that SNRT has paroxysmal episodes. In SNRT, $\mathrm{P}$ waves are similar to sinus waves. While beta-blockers are often ineffective, verapamil, diltiazem (recommended with class IIb C), and amiodarone have variable success in SNRT [25]. Catheter ablation has been proposed to be considered in drug-resistant cases in recent guidelines with class IIa C recommendation level [3, 26-28]. (3) Postural orthostatic tachycardia syndrome is defined as an increase in heart rate of $\geq 30 \mathrm{bpm}$ when standing for $>30 \mathrm{~s}$ in the absence of orthostatic hypotension. Most patients are seen between the ages of $15-25$ years and $>75 \%$ are female. Half of them recover from postural orthostatic tachycardia syndrome within 1-3 years. Regular and progressive exercise is recommended in the 2019 guidelines with class IIa B and head-up tilt sleep and compression stocking are not mentioned. Water and sodium chloride intake and ivabradine are recommended with class IIb C, while midodrine, low-dose non-selective betablocker, or pyridostigmine are recommended with class IIb B. Intravenous saline, low-dose propranolol, non-selective beta-blockers, and pyridostigmine are also mentioned in recent guidelines. Fludrocortisone and bisoprolol were recommended with class IIa, and clonidine, midodrine, methylphenidate, fluoxetine, erythropoietin, ergotamine, and phenobarbitone had class IIb recommendations in previous guidelines [1].

Focal AT. An organised atrial rhythm $>100 \mathrm{bpm}$ and a focus spreading both atria centrifugally is the definition of focal AT. In haemodynamically unstable patients, syn- chronised DC cardioversion is recommended with class I $\mathrm{B}$. As the first approach, adenosine is recommended with class IIa B in haemodynamically stable patients with acute-onset AT. If adenosine fails, beta-blockers are recommended with class IIa C in the absence of decompensated HF and with equal suggestion, verapamil and diltiazem are recommended with class IIa $\mathrm{C}$ in the absence of hypotension or HFrEF. If these drugs are ineffective, intravenous ibutilide, flecainide, propafenone, or amiodarone may be used with class IIb C. Ibutilide was not mentioned in previous guidelines; furthermore, digoxin had class IIb $\mathrm{C}$ and flecainide, propafenone, procainamide, amiodarone, and sotalol had class IIa $\mathrm{C}$ recommendation levels in previous guidelines [1]. In chronic settings, catheter ablation has maintained its level of recommendation with class I B. Catheter ablation has importance in incessant focal ATs or focal ATs causing tachycardia-induced cardiomyopathy (TCM). If ablation is not desirable or feasible or there is no incessancy of AT, beta-blockers, non-dihydropyridine calcium channel blockers (in the absence of HFrEF), propafenone, or flecainide (in the absence of structural or ischaemic heart disease) are recommended with class IIa C. If all of the above fail, as a new recommendation with class IIb C, ivabradine with beta-blocker is a new choice. If this option also fails, amiodarone is conceivable with class IIb C. In previous guidelines, beta-blockers and calcium channel blockers had class I C and flecainide, sotalol, disopyramide, and amiodarone had class IIa $\mathrm{C}$ recommendation levels [1].

Multifocal AT. An irregular rapid rhythm with at least three distinct morphologies of $\mathrm{P}$ waves is defined as multifocal AT. The prevalence of multifocal AT is about $<1 \%$ in hospitalised patients. This rhythm appears more often in patients with pulmonary diseases. First-line treatment improves the underlying conditions if it is feasible (class I C). Intravenous magnesium may be helpful even in case of normal serum magnesium levels [29]. Intravenous beta-blockers or intravenous non-dihydropyridine calcium channel blockers are recommended with class IIa B in acute settings. In chronic settings, oral verapamil, diltiazem (exceptionally in HFrEF), or beta-blockers are recommended with class IIa B in recurrent symptomatic multifocal AT. In a study metoprolol has been found to be superior to verapamil [30], and beta-blocker therapy was considered as contraindicated in previous guidelines [1]. If drug therapy is ineffective and LV dysfunction develops, AV nodal ablation followed by pacing, preferably biventricular or His bundle pacing, is recommended with class IIa C. 


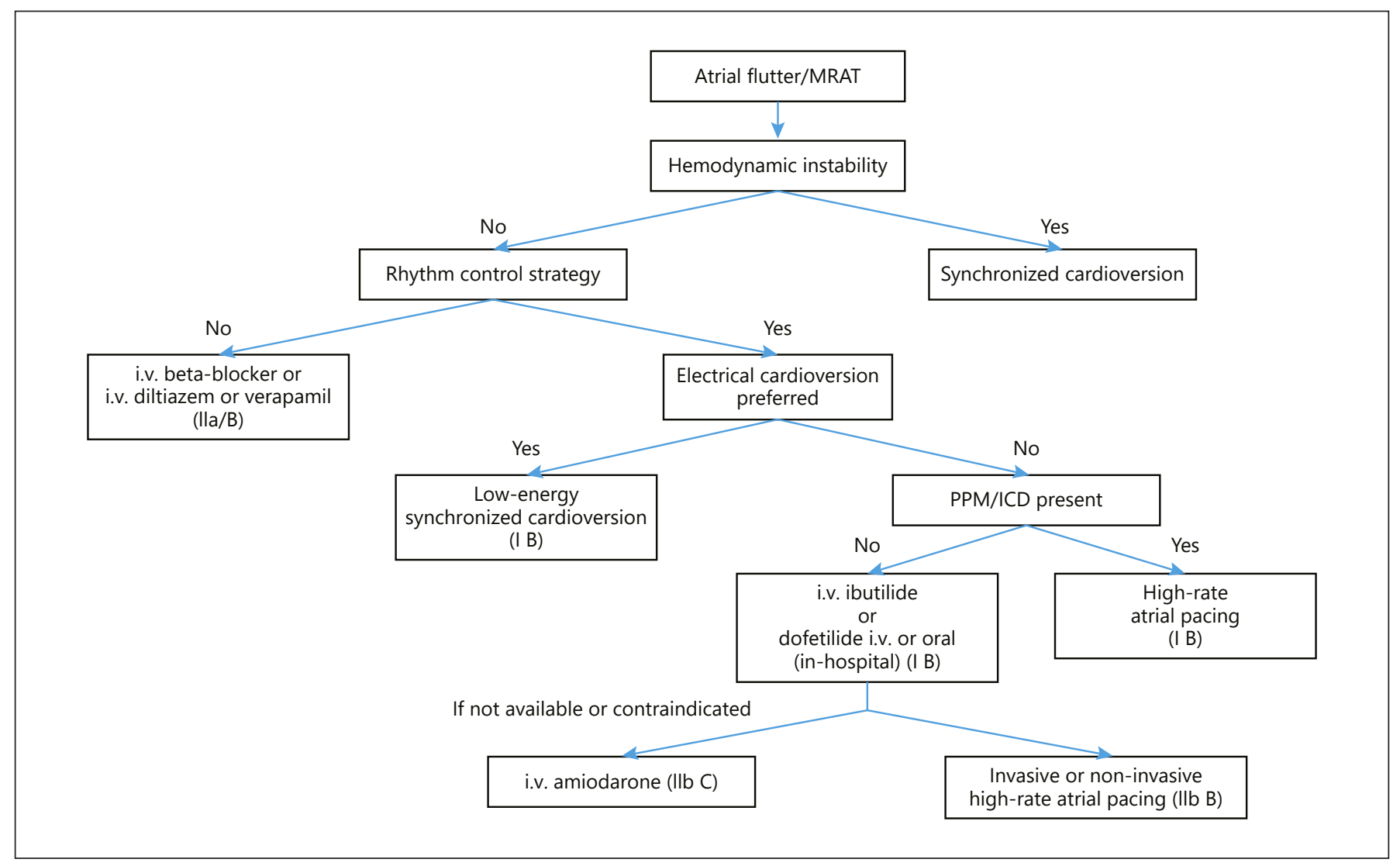

Fig. 2. Acute therapy of stable atrial flutter or macro-re-entrant AT (MRAT). AT, atrial tachycardia; i.v., intravenous; ICD, implantable cardioverter defibrillator; PPM, permanent pacemaker.

Macro-Re-Entrant AT. Definitely, the most detailed subject is typical atrial flutter, under the cavotricuspid isthmus (CTI)-dependent macro-re-entrant AT subsection of recent guidelines [3]. The typical atrial flutter manifests in two ways: common (counter-clockwise) and reverse (clockwise). In typical common atrial flutter, the activation uses the CTI as an inferior boundary. Typical saw-tooth waves are observed as negative in inferior leads and positive in V1. Anticoagulation is recommended with class I B in patients with atrial flutter concomitant AF. If patients have atrial flutter and do not have AF, anticoagulation is recommended with class IIa C, but the arrhythmia duration threshold is not clearly defined. In acute therapy, synchronised DC cardioversion is recommended with class I B in haemodynamically unstable patients. Adenosine can reveal the flutter waves, but it can produce a rebound increase in AV conduction and therefore should only be used if it is necessary for diagnosis and if resuscitation equipment is available. Anticoagulation before cardioversion in atrial flutter should be performed as in AF patients [31, 32]. Class Ic antiarrhythmics (propafenone and flecainide) are not recommended in patients with atrial flutter for conversion to sinus rhythm because slowing of the atrial rate may result in $1: 1 \mathrm{AV}$ conduction (class III B). Recommendations for acute treatment of atrial flutter/macro-re-entrant AT are presented in Figure 2. In chronic therapy, catheter ablation is the first choice of therapy. It is recommended in patients who have symptomatic, recurrent episodes of CTIdependent flutter with class I A and class I B in symptomatic and recurrent episodes with non-CTI-dependent AT in experienced centres. As in previous guidelines, after the first episode of atrial flutter, catheter ablation is recommended with class IIa B. Newly in the 2019 guidelines, catheter ablation is recommended with class I B in patients with LV systolic dysfunction due to TCM or persistent atrial flutter. Beta-blockers and non-dihydropyridine calcium channel blockers are recommended with class IIa $\mathrm{C}$, and if any of the previous fails, amiodarone may be considered to maintain sinus rhythm with class 


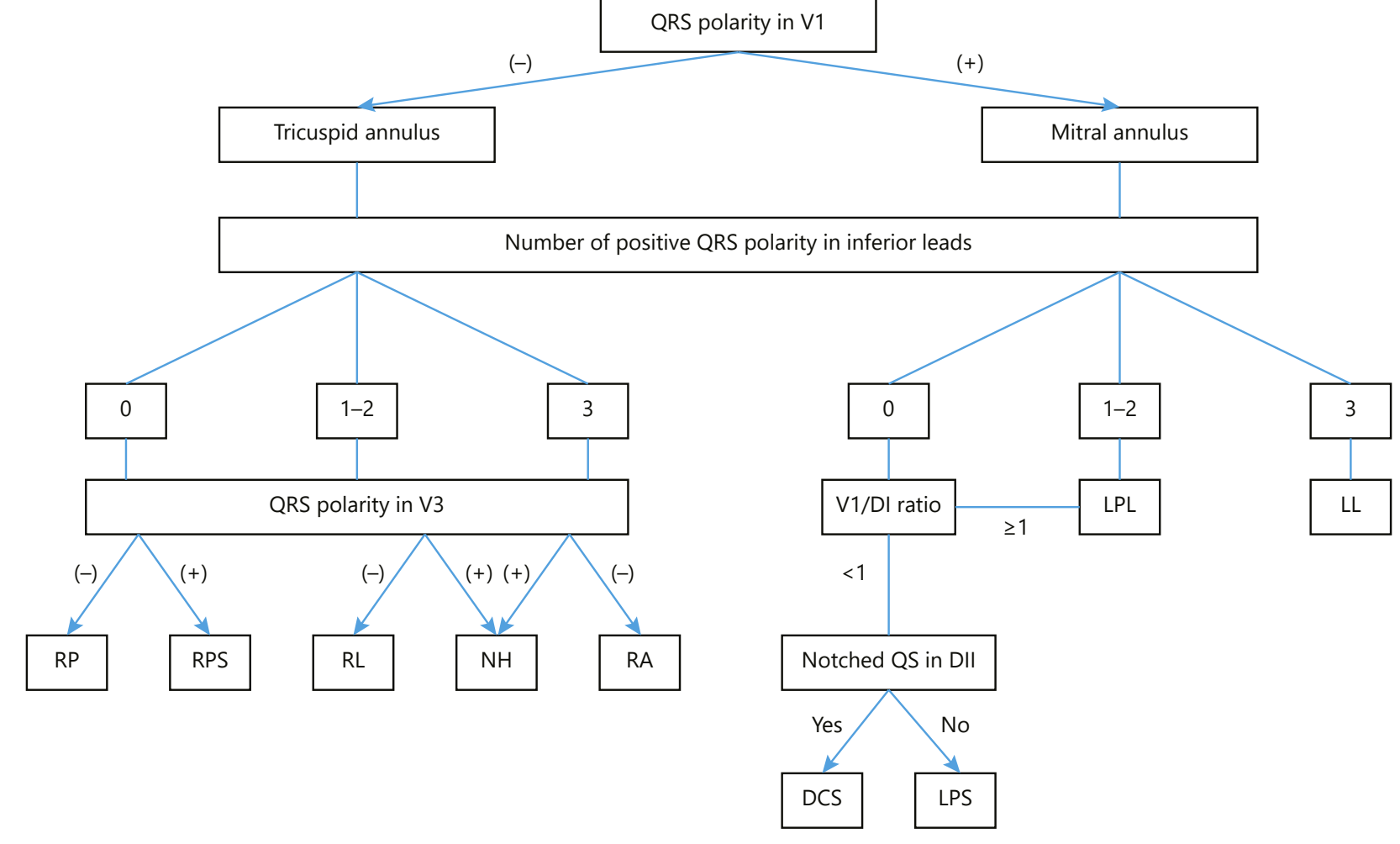

Fig. 3. AP layout in the presence of maximum pre-excitation. AP, accessory pathway; DCS, deep coronary sinus; LL, left lateral; LPL, left posterolateral; LPS, left paraseptal; NH, nodo-Hisian; RA, right anterior; RL, right lateral; RP, right posterior; RPS, right paraseptal.

IIb C. The ablate and pace method is recommended with class IIa C, if all the other approaches fail and the patient has symptomatic macro re-entrant arrhythmias with fast ventricular rate.

\section{AV Junctional Arrhythmias}

AVNRT. The AV node is a three-dimensional structure which is considered a structure consisting of two different conduction paths, a slow and a fast pathway. Slow and fast pathways may vary as anterograde or retrograde, which is the reason for the classification of AVNRT into the forms of slow-fast, fast-slow, or slow-slow. All tachycardia forms of AVNRT are evaluated within this comprehensive model. AVNRT attacks may occur early in life or later (in the fourth or fifth decades of life) [33]. About half of the patients have minimal symptoms with shortlived tachycardia [34]. AVNRT usually presents with a narrow QRS complex, and if there is an aberrancy, it usually tends to be of the right bundle branch block type. In typical forms of AVNRT, i.e., slow-fast AVNRT, the ret- rograde $\mathrm{P}$ waves are seen close to the $\mathrm{QRS}$ complex or are undetectable (pseudo R deflection in lead V1 and pseudo $S$ wave in inferior leads). In the atypical form of AVNRT, the RP interval is longer than the PR interval and $\mathrm{P}$ waves are clearly visible. In haemodynamically unstable patients with AVNRT, synchronised DC cardioversion is indicated (class I B). In haemodynamically stable patients, vagal manoeuvres and adenosine are recommended (class I B). If these approaches fail, beta-blockers are recommended with class IIa $\mathrm{C}$, or non-dihydropyridine calcium channel blockers are recommended with class IIa B to control or convert the AVNRT. If drug therapy fails, synchronised $\mathrm{DC}$ cardioversion is recommended (class I B). As in previous guidelines, catheter ablation is recommended with class I B for symptomatic, recurrent AVNRT. In a recent randomised clinical trial, catheter ablation was compared to drug therapy, and significant benefits were found in arrhythmia-related hospitalisations with catheter ablation [35]. The slow-pathway modification combined with ablation lesions at the inferior part of the triangle of Koch 
has a success rate of $97 \%$, lower recurrence rates with $1.3-$ $4 \%$, and an AV block risk $<1 \%$ [36-39]. If cryoablation is performed, it carries a lower risk of AV block, but has higher recurrence rates. For asymptomatic and non-recurrent AVNRT, catheter ablation is recommended if drug therapy is not considered. If catheter ablation is not considered, diltiazem, verapamil, beta-blocker, or diltiazem plus beta-blocker are recommended with class IIa B. In previous guidelines, diltiazem, verapamil, and betablockers had class I recommendation levels. In this regard, the recent guideline has simpler and clearer recommendations on chronic therapy. Since there are shortlived and infrequent episodes in half of the patients, abstinence from therapy should be considered in these patients (class IIa C) [34]. Although flecainide is mentioned in the recent guidelines, it has no class of recommendation. In addition, propafenone, amiodarone, sotalol, digoxin, and the pill-in-the-pocket approach are not longer mentioned [3].

Non-Re-Entrant Junctional Tachycardia. Junctional ectopic tachycardia or focal tachycardia is a tachycardia caused by abnormal automaticity in the AV node or proximal His bundle. In acute therapy, propranolol, verapamil, procainamide, or flecainide can be used, although the data are limited. The congenital junctional tachycardia form has morbidity and mortality risk, and amiodarone alone or with propafenone or ivabradine can be used. Catheter ablation carries a higher AV block risk and a low success rate compared to AVNRT. Cryoablation is safer in such a situation $[40,41]$. Non-paroxysmal junctional tachycardia was diagnosed as junctional tachycardia in the past and was considered as for example digitalis-induced delayed after depolarisations and triggered activity in the AV node.

\section{AV Arrhythmias}

AVRT uses the AV node-His-Purkinje system and an AP called bypass tract. The circuit rarely consists of two APs (pathway-to-pathway conduction). APs are made up of single or multiple conductive cells that bypass the physiologic system and connect the atrial and ventricular myocardium [42]. Sixty percent of APs are found along the mitral valve (left free wall), $25 \%$ of them are located at the septal aspect of the mitral or tricuspid annulus, and $15 \%$ are located at the right atrial free wall [43-45]. If an AP conducts as anterograde, pre-excitation is referred to as manifest because ventricular pre-excitation is visible at rest. On the other hand, if an AP conducts as retrograde, the ventricular pre-excitation is not detectable at rest, and thus it referred to as concealed. WPW syndrome is a con-

ESC 2019 Supraventricular Tachycardia Guidelines: Review dition that presents with manifestations of AP and recurrent arrhythmias. WPW syndrome has characteristics of resting ECG which are short PR interval, a delta wave, and wide QRS complexes. Figure 3 provides an algorithm based on maximal pre-excitation to localise the AP in adults [46].

About $90 \%$ of AVRTs are orthodromic AVRTs. The re-entrant impulse conducts from the atrium to the ventricle using the AV node-His-Purkinje system and turn back to the atrium from the ventricle via the AP. Orthodromic AVRTs are rapid tachycardias with frequencies ranging from 150 to $220 \mathrm{bpm}$. During the tachycardia, the $\mathrm{RP}$ interval is usually constant, QRS may be narrow, functional bundle branch block may be present (usually on the same side as an AP), or ST segment may be depressed.

Antidromic AVRT occurs in 3-8\% of patients with WPW syndrome. Multiple APs may be detected in 30$60 \%$ of patients. In this type of AVRT, QRS complexes are widened and it is difficult to evaluate the RP interval since the $\mathrm{P}$ wave is usually not easily detected within the ST-T segment in ECG.

Pre-excited AF has been found in 50\% of WPW patients, and these tend to be younger and to have no structural heart diseases. If AP has a short anterograde refractory period, this condition is life-threatening due to risk of ventricular fibrillation (VF).

Permanent junctional reciprocating tachycardia is concealed AVRT that needs careful consideration as it causes TCM, especially in young patients. In ECG, deeply inverted retrograde $\mathrm{P}$ waves in inferior leads are seen. Catheter ablation is strongly recommended in symptomatic patients or inpatients with TCM.

In haemodynamically unstable patients, synchronised $\mathrm{DC}$ cardioversion is recommended with class I B in AVRT or pre-excited AF. In stable patients with orthodromic AVRT, vagal manoeuvres are recommended first, and if this fails, adenosine is recommended (class I B). Adenosine should be used with caution due to potential induction of rapid ventricular rate $\mathrm{AF}$ and risk of VF. If adenosine fails in orthodromic AVRT, intravenous verapamil, diltiazem (in the absence of HFrEF), or intravenous betablocker such as esmolol or metoprolol (in the absence of decompensated HF) should be considered with class IIa $\mathrm{B}$ and class IIa C, respectively. In antidromic AVRT, if vagal manoeuvres and adenosine fail, intravenous ibutilide, procainamide, intravenous flecainide, propafenone, or synchronised DC cardioversion should be considered with class IIa B recommendation level. In refractory cases with AVRT, amiodarone may be an option (class IIb B). In haemodynamically stable patients with pre-excited 


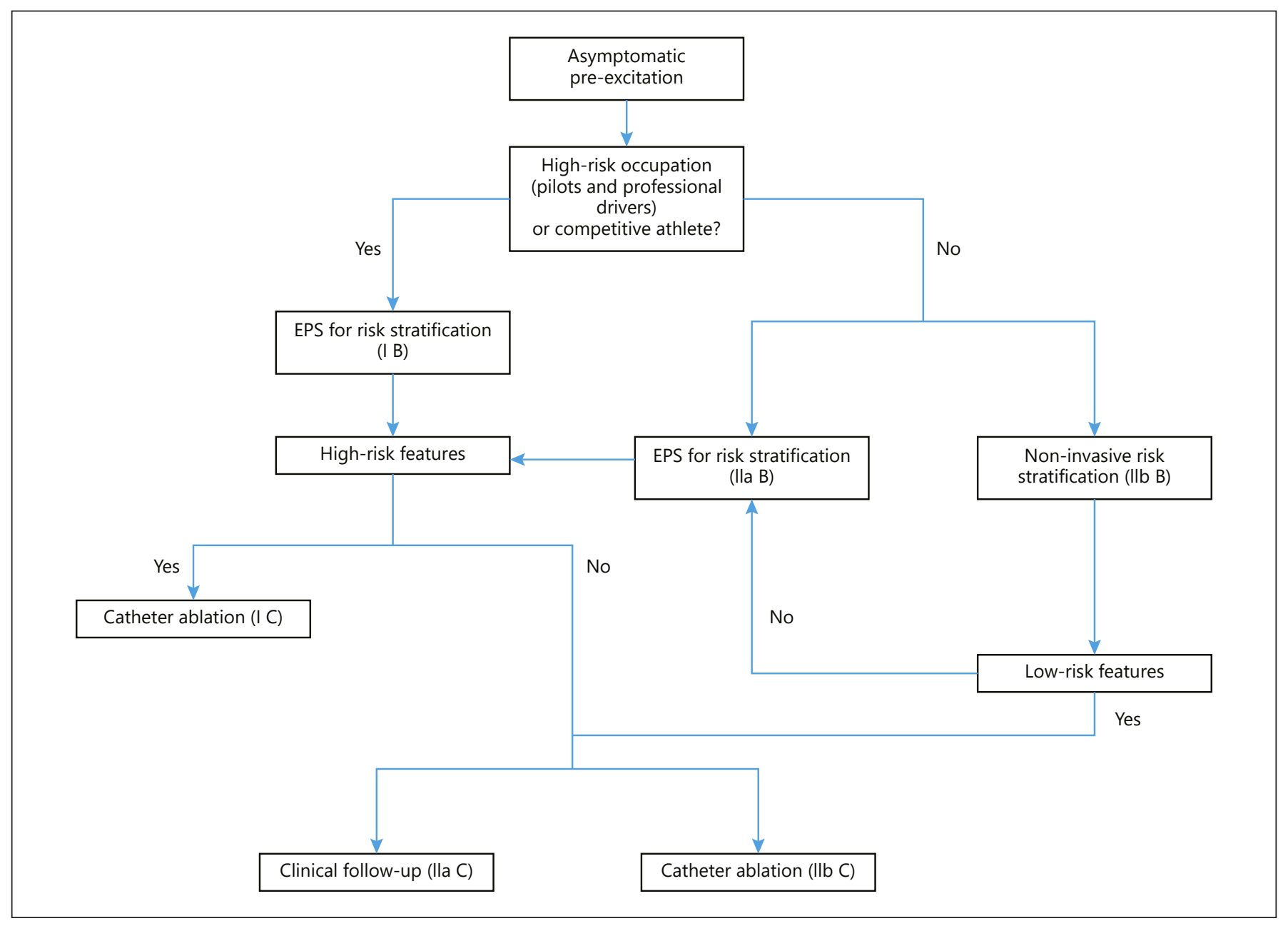

Fig. 4. The approach to asymptomatic pre-excitation. EPS, electrophysiologic study.

$\mathrm{AF}$, intravenous ibutilide or intravenous procainamide should be considered (class IIa B). Flecainide or propafenone (intravenously) are other options (class IIb B). If drug therapy fails, synchronised DC cardioversion is recommended with class I B in patients with AVRT and preexcited AF. Amiodarone carries VF risk in patients with pre-excited AF and is not recommended (class III B).

In chronic therapy of AVRT, catheter ablation is recommended with class I B in patients with symptomatic, recurrent AVRT episodes. Beta-blockers or non-dihydropyridine calcium channel blockers (in the absence of HFrEF) are conceivable with no signs of pre-excitation present in resting ECG when ablation is not desired or not practicable (class IIa B). In patients with AVRT, flecainide or propafenone may be administered with no ischaemic or structural heart disease when ablation is not desirable or feasible (class IIb C). AV node blocking agents such as verapamil, diltiazem, beta-blockers, amiodarone, and digoxin are not recommended in patients with pre-excited AVRT (class III B). In previous guidelines, flecainide, propafenone, sotalol, and amiodarone had class IIa C, beta-blockers had class IIb C, and verapamil, diltiazem, and digoxin had class III recommendation levels in poorly tolerated AVRT section. In single or infrequent episodes of AVRT, the pill-in-the-pocket approach with verapamil, diltiazem, and beta-blocker had class I B, catheter ablation had class IIa B, and sotalol, amiodarone, flecainide, and propafenone had class IIb C recommendation level in previous guidelines.

Most patients with asymptomatic pre-excited AF do not experience any clinic events in their lifetime. Clinical arrhythmia develops in only one-fifth of patients and the most common arrhythmia is AVRT with a rate of $80 \%$, followed by AF with a rate of $20-30 \%$. The risk of cardiac 
arrest and VF is 2.4 per 1,000 person-years [47]. High-risk properties for sudden cardiac death in electrophysiologic study are shortest pre-excited RR interval $\leq 250 \mathrm{~ms}$, anterograde effective refractory period of AP $\leq 250 \mathrm{~ms}$, multiple APs, and inducible AP-mediated tachycardia; catheter ablation is recommended in such patients [3]. In non-invasive testing, an abrupt and complete normalisation of PR interval and loss of delta wave during exercise testing or following the administration of propafenone, procainamide, or disopyramide has been considered a low-risk marker [48-51]. Intermittent pre-excitation on resting ECG or ambulatory monitoring is now considered an imperfect marker of low-risk AP [50, 52-56]. The approach to asymptomatic pre-excitation is summarised in Figure 4. In the new guidelines, it is proposed to discuss the risks and benefits of ablation in high-risk patients, especially the risk of heart blocks associated with anteroseptal or midseptal AP ablations (class I C). Finally, if a patient with asymptomatic AP has LV dysfunction due to electrical dyssynchrony, catheter ablation should be considered (class IIa C).

\section{Special Patient Populations}

\section{SVT in Pregnancy}

SVT is observed in 22-24 of 100,000 pregnancies, especially in the third trimester or peripartum. SVTs in pregnancy are mostly benign and can be managed with standard medical therapy, with a few exceptions. Catheter ablation is primarily recommended with class I C in symptomatic women with recurrent SVT who are planning pregnancy. If haemodynamic instability occurs with SVT in pregnant women, synchronised DC cardioversion is urgent (class I C). Vagal manoeuvres are recommended first, and if they fail, adenosine is recommended for acute conversion of SVT in pregnancy (class I C). An intravenous beta-blocker - except atenolol - should be considered for acute conversion of SVT or rate control (class IIa C). If beta-blockers fail, intravenous digoxin in the latest pocket Gls version for rate control of AT should be considered (class IIa C). In pregnant patients with atrial flutter, intravenous ibutilide in the latest pocket Gls version may be considered for termination of atrial flutter (class IIb C).

In the first trimester, avoiding all antiarrhythmic drugs, if possible, is recommended with class I C. In prevention of SVTs without WPW syndrome, beta-1 selective beta-blockers - except atenolol - or verapamil are utilisable in order of preference (class IIa C). If a pregnant

ESC 2019 Supraventricular Tachycardia Guidelines: Review woman without ischaemic or structural heart disease has SVT with WPW syndrome, flecainide or propafenone should be considered with class IIa C for prevention of SVT. When AV node blocking agents fail to prevent SVT in pregnant women without ischaemic or structural heart disease, flecainide and propafenone should also be considered with class II a C. In pregnant women without WPW syndrome, digoxin or verapamil should be considered for rate control of AT if beta-blockers fail (class IIa $\mathrm{C})$. Amiodarone is harmful and not recommended in pregnant women (class III C). Fluoroless catheter ablation provides convenience for pregnant women. This approach should be considered in experienced centres for those who cannot tolerate SVT or who have drug-refractory SVT (class IIa C).

\section{Tachycardia-Induced Cardiomyopathy}

Persistence of SVT or VT can cause reversible cardiomyopathies and this is defined as TCM $[3,57]$. Although this syndrome was initially described with permanent junctional reciprocating tachycardia, incessant AVRTs due to septal APs, ATs, or rapid AFs can cause TCM. In any patient, when new-onset LV dysfunction is observed, TCM should be considered among the aetiology. The diagnosis is based on exclusion of other causes of cardiomyopathy and demonstrating LV function recovery after controlling the tachycardia. Cardiac magnetic resonance is a convenient imaging modality to evaluate intrinsic structure.

Catheter ablation is recommended and necessary in patients with TCM due to SVT (class I B). If catheter ablation fails or is not applicable, beta-blockers are recommended with class IA recommendation level for TCM due to SVT. In a patient with reduced LV ejection fraction with a heart rate $>100 \mathrm{bpm}$, it is recommended to consider TCM (class I B). Twenty-four-hour or multiday ambulatory ECG monitoring should be considered to identify subclinical or intermittent arrhythmias for the diagnosis of TCM (class IIa B). If TCM cannot be ablated or controlled by drugs, ablate and pace is recommended as a last resort (class I C).

\section{SVT in Sports}

Athletes with SVTs should be evaluated for presence of underlying cardiac disease, thyroid dysfunction, electrolyte imbalance, and use of stimulants or performanceenhancing drugs. WPW syndrome is one of the reasons of sudden cardiac death in young individuals and athletes. If an athlete has symptomatic WPW syndrome (with AVRT or AF), catheter ablation of the AP is 
mandatory (class of recommendation not specified). In asymptomatic athletes with ventricular pre-excitation, invasive risk stratification should be performed and ablation is mandatory in patients at high risk. Sports are allowed 1 month after ablation if there are no recurrences. Asymptomatic patients with low risk are allowed to practice competitive sports. In athletes with AVNRT, orthodromic AVRT over concealed AP, and AT, catheter ablation should be recommended (class of recommendation not specified). If an athlete with SVT does not wish to undergo catheter ablation or catheter ablation fails or is not feasible and the arrhythmia is sporadic, unrelated to the cardiac disease, and well tolerated, the athlete can compete in sports without intrinsic risk of unconsciousness (such as drivers, pilots, and horse riders) (no class of recommendation).

\section{Conclusion}

The recent SVT guidelines of the ESC are a team work covering the literature extensively and offer a simpler and more comprehensive approach to clinicians with a va- riety of algorithms. Although current knowledge, from cases to randomised clinical trials, causes some recommendation level changes in drug treatments, the effect of catheter ablation in chronic therapy has increased significantly. Current and new studies will contribute to the increase in knowledge on SVTs.

\section{Conflict of Interest Statement}

The authors have no conflicts of interest to disclose.

\section{Funding Sources}

There were no funding sources.

\section{Author Contributions}

Concept: B. Görenek, T. Ulus. Design: E. Babayiğit, T. Ulus, B. Görenek. Supervision: E. Babayiğit, T. Ulus, B. Görenek. Data collection and/or processing: E. Babayiğit, T. Ulus, B. Görenek. Analysis and/or interpretation: E. Babayiğit, T. Ulus, B. Görenek. Literature search: E. Babayiğit, T. Ulus. Writing: E. Babayiğit, T. Ulus. Critical review: B. Görenek, T. Ulus.

\section{References}

1 Blomström-Lundqvist C, Scheinman MM, Aliot EM, Alpert JS, Calkins H, Camm AJ, et al.; European Society of Cardiology Committee, NASPE-Heart Rhythm Society. ACC/AHA/ ESC guidelines for the management of patients with supraventricular arrhythmias - executive summary. A report of the American College of Cardiology/American Heart Association task force on practice guidelines and the European Society of Cardiology committee for practice guidelines (writing committee to develop guidelines for the management of patients with supraventricular arrhythmias) developed in collaboration with NASPE-Heart Rhythm Society. J Am Coll Cardiol. 2003 Oct;42(8):1493-531.

2 Page RL, Joglar JA, Caldwell MA, Calkins H, Conti JB, Deal BJ, et al. 2015 ACC/AHA/HRS Guideline for the Management of Adult Patients With Supraventricular Tachycardia: A Report of the American College of Cardiology/American Heart Association Task Force on Clinical Practice Guidelines and the Heart Rhythm Society. J Am Coll Cardiol. 2016 Apr;67(13):e27-115.

3 Brugada J, Katritsis DG, Arbelo E, Arribas F, Bax JJ, Blomström-Lundqvist C, et al.; ESC Scientific Document Group. 2019 ESC Guidelines for the management of patients with supraventricular tachycardia. The Task Force for the management of patients with supraventricular tachycardia of the European Society of Cardiology (ESC). Eur Heart J. 2020 Feb;41(5):655-720.
4 Orejarena LA, Vidaillet H Jr, DeStefano F, Nordstrom DL, Vierkant RA, Smith PN, et al. Paroxysmal supraventricular tachycardia in the general population. J Am Coll Cardiol. 1998 Jan;31(1):150-7.

5 Wu MH, Chen HC, Kao FY, Huang SK. Postnatal cumulative incidence of supraventricular tachycardia in a general pediatric population: A national birth cohort database study. Heart Rhythm. 2016 Oct;13(10):2070-5.

6 García-Fernández FJ, Ibáñez Criado JL, Quesada Dorador A, Álvarez-López M, Almendral J, Alonso C, et al.; collaborators of the Spanish Catheter Ablation Registry; REGISTRY COLLABORATORS. Spanish Catheter Ablation Registry. 17th Official Report of the Spanish Society of Cardiology Working Group on Electrophysiology and Arrhythmias (2017). Rev Esp Cardiol (Engl Ed). 2018 Nov;71(11):941-51.

7 Hosseini SM, Rozen G, Saleh A, Vaid J, Biton Y, Moazzami K, et al. Catheter ablation for cardiac arrhythmias: utilization and in-hospital complications, 2000 to 2013. JACC Clin Electrophysiol. 2017 Nov;3(11):1240-8.

8 Holmqvist F, Kesek M, Englund A, BlomströmLundqvist C, Karlsson LO, Kennebäck G, et al. A decade of catheter ablation of cardiac arrhythmias in Sweden: ablation practices and outcomes. Eur Heart J. 2019 Mar;40(10):820-30.

9 Chang SH, Kuo CF, Chou IJ, See LC, Yu KH, Luo SF, et al. Outcomes associated with parox- ysmal supraventricular tachycardia during pregnancy. Circulation. $2017 \mathrm{Feb}$;135(6):616-8.

10 Krahn AD, Manfreda J, Tate RB, Mathewson FA, Cuddy TE. The natural history of electrocardiographic preexcitation in men. The Manitoba Follow-up Study. Ann Intern Med. 1992 Mar;116(6):456-60.

11 Granada J, Uribe W, Chyou PH, Maassen K, Vierkant R, Smith PN, et al. Incidence and predictors of atrial flutter in the general population. J Am Coll Cardiol. 2000 Dec;36(7):2242-6.

12 Page RL, Wilkinson WE, Clair WK, McCarthy EA, Pritchett EL. Asymptomatic arrhythmias in patients with symptomatic paroxysmal atrial fibrillation and paroxysmal supraventricular tachycardia. Circulation. 1994 Jan;89(1):224-7.

13 Thavendiranathan P, Bagai A, Khoo C, Dorian P, Choudhry NK. Does this patient with palpitations have a cardiac arrhythmia? JAMA. 2009 Nov;302(19):2135-43.

14 Skov MW, Rasmussen PV, Ghouse J, Hansen SM, Graff C, Olesen MS, et al. Electrocardiographic preexcitation and risk of cardiovascular morbidity and mortality. Results from the Copenhagen ECG Study. Circ Arrhythm Electrophysiol. 2017 Jun;10(6):e004778.

15 Chinen S, Miura M, Tamame T, Matsuoka M, Ohki H, Sumitomo N. Life-threatening atrial tachycardia after the Senning operation in a patient with transposition of the great arteries. Heart Vessels. 2012 Jul;27(4):424-7. 
16 Knuuti J, Wijns W, Saraste A, Capodanno D, Barbato E, Funck-Brentano C, et al.; ESC Scientific Document Group. 2019 ESC Guidelines for the diagnosis and management of chronic coronary syndromes. Eur Heart J. $2020 \mathrm{Jan} ; 41(3): 407-77$.

17 Roberts-Thomson KC, Kistler PM, Kalman JM. Focal atrial tachycardia I: clinical features, diagnosis, mechanisms, and anatomic location. Pacing Clin Electrophysiol. 2006 Jun;29(6):643-52.

18 Alzand BS, Crijns HJ. Diagnostic criteria of broad QRS complex tachycardia: decades of evolution. Europace. 2011 Apr;13(4):465-72.

19 Burki NK, Dale WJ, Lee LY. Intravenous adenosine and dyspnea in humans. J Appl Physiol (1985). 2005 Jan;98(1):180-5.

20 Camm AJ, Garratt CJ. Adenosine and supraventricular tachycardia. N Engl J Med. 1991 Dec;325(23):1621-9.

21 Turley AJ, Murray S, Thambyrajah J. Pre-excited atrial fibrillation triggered by intravenous adenosine: a commonly used drug with potentially life-threatening adverse effects. Emerg Med J. 2008 Jan;25(1):46-8.

22 Stambler BS, Dorian P, Sager PT, Wight D, Douville P, Potvin D, et al. Etripamil nasal spray for rapid conversion of supraventricular tachycardia to sinus rhythm. J Am Coll Cardiol. 2018 Jul;72(5):489-97.

23 Ortiz M, Martín A, Arribas F, Coll-Vinent B, Del Arco C, Peinado R, et al.; PROCAMIO Study Investigators. Randomized comparison of intravenous procainamide vs. intravenous amiodarone for the acute treatment of tolerated wide QRS tachycardia: the PROCAMIO study. Eur Heart J. 2017 May;38(17):1329-35.

24 Olshansky B, Sullivan RM. Inappropriate sinus tachycardia. Europace. 2019 Feb;21(2):194-207.

25 Gomes JA, Hariman RJ, Kang PS, Chowdry IH. Sustained symptomatic sinus node reentrant tachycardia: incidence, clinical significance, electrophysiologic observations and the effects of antiarrhythmic agents. J Am Coll Cardiol. 1985 Jan;5(1):45-57.

26 Malik AK, Ching CK, Liew R, Chong DT, Teo WS. Successful ablation of sinus node reentrant tachycardia using remote magnetic navigation system. Europace. 2012 Mar;14(3):455-6.

27 Cossú SF, Steinberg JS. Supraventricular tachyarrhythmias involving the sinus node: clinical and electrophysiologic characteristics. Prog Cardiovasc Dis. 1998 Jul-Aug;41(1):51-63.

28 Sanders WE Jr, Sorrentino RA, Greenfield RA, Shenasa H, Hamer ME, Wharton JM. Catheter ablation of sinoatrial node reentrant tachycardia. J Am Coll Cardiol. 1994 Mar;23(4):926-34.

29 Iseri LT, Fairshter RD, Hardemann JL, Brodsky MA. Magnesium and potassium therapy in multifocal atrial tachycardia. Am Heart J. 1985 Oct;110(4):789-94.

30 Arsura E, Lefkin AS, Scher DL, Solar M, Tessler S. A randomized, double-blind, placebocontrolled study of verapamil and metoprolol in treatment of multifocal atrial tachycardia. Am J Med. 1988 Oct;85(4):519-24.

31 Kirchhof P, Benussi S, Kotecha D, Ahlsson A, Atar D, Casadei B, et al.; ESC Scientific Docu- ment Group. 2016 ESC Guidelines for the management of atrial fibrillation developed in collaboration with EACTS. Eur Heart J. 2016 Oct;37(38):2893-962.

32 Katritsis DG, Josephson ME. Anticoagulation for cardioversion of acute onset atrial fibrillation: time to revise guidelines? JACC Clin Electrophysiol. 2016 Aug;2(4):495-7.

33 Pentinga ML, Meeder JG, Crijns HJ, de Muinck ED, Wiesfeld AC, Lie KI. Late onset atrioventricular nodal tachycardia. Int J Cardiol. 1993 Mar;38(3):293-8.

34 D’Este D, Zoppo F, Bertaglia E, Zerbo F, Picciolo A, Scarabeo V, et al. Long-term outcome of patients with atrioventricular node reentrant tachycardia. Int J Cardiol. 2007 Feb; 115(3):350-3.

35 Katritsis DG, Zografos T, Katritsis GD, Giazitzoglou E, Vachliotis V, Paxinos G, et al. Catheter ablation vs. antiarrhythmic drug therapy in patients with symptomatic atrioventricular nodal re-entrant tachycardia: a randomized, controlled trial. Europace. 2017 Apr;19(4):602-6.

36 Spector P, Reynolds MR, Calkins H, Sondhi M, $\mathrm{Xu}$ Y, Martin A, et al. Meta-analysis of ablation of atrial flutter and supraventricular tachycardia. Am J Cardiol. 2009 Sep;104(5):671-7.

37 Bohnen M, Stevenson WG, Tedrow UB, Michaud GF, John RM, Epstein LM, et al. Incidence and predictors of major complications from contemporary catheter ablation to treat cardiac arrhythmias. Heart Rhythm. 2011 Nov;8(11):1661-6.

38 Morady F. Catheter ablation of supraventricular arrhythmias: state of the art. Heart Rhythm. 2004 Nov;1(5 Suppl):67C-84C.

39 Van Hare GF, Javitz H, Carmelli D, Saul JP, Tanel RE, Fischbach PS, et al.; Pediatric Electrophysiology Society. Prospective assessment after pediatric cardiac ablation: demographics, medical profiles, and initial outcomes. J Cardiovasc Electrophysiol. 2004 Jul;15(7):759-70.

40 Entenmann A, Michel M, Herberg U, Haas N, Kumpf M, Gass M, et al. Management of postoperative junctional ectopic tachycardia in pediatric patients: a survey of 30 centers in Germany, Austria, and Switzerland. Eur J Pediatr. 2017 Sep;176(9):1217-26.

41 Law IH, Von Bergen NH, Gingerich JC, Saarel EV, Fischbach PS, Dick M 2nd. Transcatheter cryothermal ablation of junctional ectopic tachycardia in the normal heart. Heart Rhythm. 2006 Aug;3(8):903-7.

42 Ho SY. Accessory atrioventricular pathways: getting to the origins. Circulation. 2008 Mar; 117(12):1502-4.

43 Jackman WM, Wang XZ, Friday KJ, Roman CA, Moulton KP, Beckman KJ, et al. Catheter ablation of accessory atrioventricular pathways (Wolff-Parkinson-White syndrome) by radiofrequency current. N Engl J Med. 1991 Jun;324(23):1605-11.

44 Katritsis D, Bashir Y, Heald S, Poloniecki J, Ward DE. Radiofrequency ablation of accessory pathways: implications of accumulated experience and time dedicated to procedures. Eur Heart J. 1994 Mar;15(3):339-44.
45 Schlüter M, Geiger M, Siebels J, Duckeck W, Kuck KH. Catheter ablation using radiofrequency current to cure symptomatic patients with tachyarrhythmias related to an accessory atrioventricular pathway. Circulation. 1991 Oct;84(4):1644-61.

46 Pambrun T, El Bouazzaoui R, Combes N, Combes S, Sousa P, Le Bloa M, et al. Maximal pre-excitation based algorithm for localization of manifest accessory pathways in adults. JACC Clin Electrophysiol. 2018 Aug;4(8):1052-61.

47 Pappone C, Vicedomini G, Manguso F, Saviano M, Baldi M, Pappone A, et al. Wolff-Parkinson-White syndrome in the era of catheter ablation: insights from a registry study of 2169 patients. Circulation. 2014 Sep;130(10):811-9.

48 Sharma AD, Yee R, Guiraudon G, Klein GJ. Sensitivity and specificity of invasive and noninvasive testing for risk of sudden death in Wolff-Parkinson-White syndrome. J Am Coll Cardiol. 1987 Aug;10(2):373-81.

49 Daubert C, Ollitrault J, Descaves C, Mabo P, Ritter P, Gouffault J. Failure of the exercise test to predict the anterograde refractory period of the accessory pathway in Wolff Parkinson White syndrome. Pacing Clin Electrophysiol. 1988 Aug;11(8):1130-8

50 Wackel P, Irving C, Webber S, Beerman L, Arora G. Risk stratification in Wolff-Parkinson-White syndrome: the correlation between noninvasive and invasive testing in pediatric patients. Pacing Clin Electrophysiol. 2012 Dec;35(12):1451-7.

51 Gaita F, Giustetto C, Riccardi R, Mangiardi L, Brusca A. Stress and pharmacologic tests as methods to identify patients with Wolff-Parkinson-White syndrome at risk of sudden death. Am J Cardiol. 1989 Sep;64(8):487-90.

52 Gemma LW, Steinberg LA, Prystowsky EN, Padanilam BJ. Development of rapid preexcited ventricular response to atrial fibrillation in a patient with intermittent preexcitation. J Cardiovasc Electrophysiol. 2013 Mar;24(3):347-50.

53 Jastrzębski M, Kukla P, Pitak M, Rudziński A, Baranchuk A, Czarnecka D. Intermittent preexcitation indicates "a low-risk" accessory pathway: time for a paradigm shift? Ann Noninvasive Electrocardiol. 2017 Nov;22(6):e12464.

54 Cohen M. Intermittent preexcitation: should we rethink the current guidelines? Pacing Clin Electrophysiol. 2016 Jan;39(1):9-11.

55 Mah DY, Sherwin ED, Alexander ME, Cecchin F, Abrams DJ, Walsh EP, et al. The electrophysiological characteristics of accessory pathways in pediatric patients with intermittent preexcitation. Pacing Clin Electrophysiol. 2013 Sep;36(9):1117-22.

56 Spar DS, Silver ES, Hordof AJ, Liberman L. Relation of the utility of exercise testing for risk assessment in pediatric patients with ventricular preexcitation to pathway location. Am J Cardiol. 2012 Apr;109(7):1011-4.

57 Ulus T, Okyay K, Kabul HK, Özcan EE, Özeke Ö, Altay H, et al. Turkish Society of Cardiology consensus paper on management of arrhythmia-induced cardiomyopathy. Anatol J Cardiol. 2019 Feb;21(2):98-106. 